\title{
Recognition failure, associative relatedness, and recognition memory
}

\author{
DAVID L. HORTON and TIMOTHY J. PAVLICK \\ University of Maryland, College Park, Maryland
}

\begin{abstract}
The encoding-specificity recognition-failure paradigm was employed in four experiments. The materials presented during encoding were identical in all four. These word pairs varied in associative relatedness in an asymmetrical manner. They also varied in level of associative relatedness. As expected, the results showed substantial amounts of recognition failure when the dominant direction of associative relatedness went from cue to target. Very little recognition failure was observed in the target-to-cue associative relatedness condition. These findings are discussed in the context of factors leading to the phenomenon of recognition failure and to the relationship between recall and recognition.
\end{abstract}

The recognition failure paradigm has been employed by students of memory for at least two decades. Typically in this paradigm, word pairs are presented during study; usually one member of the pair is presented in lowercase (the cue or context word), and the other, in uppercase (the target word). Following pair presentation, a recognition memory test is presented; this is followed by a cued recall task in which the cue words are presented and recall of the target words is requested. Recognition failure is observed when items that were not recognized are subsequently recalled.

This paradigm has been employed for a variety of purposes. Initially, for example, the recognition failure phenomenon suggested implications about the generationrecognition hypothesis (Kintsch, 1970), according to which recall should never exceed recognition because recall involves both generation and recognition, whereas recognition is a unitary process. But the empirical literature clearly indicates that the phenomenon of recognition failure of subsequently recallable items is reliable (see Tulving, 1983).

The phenomenon of recognition failure also has implications for the nature of recognition memory, which is the primary focus of the present paper. George Mandler and his associates (Mandler, 1980; Rabinowitz, Mandler, \& Barsalou, 1977) claim that recognition involves two processes: perceptual familiarity and contextual retrieval. Rabinowitz et al. explain recognition failure in terms of retrieval asymmetry: when the cue word elicits the target word in recall but did not do so during the recognition test, recognition failure has occurred; this happens because the target word was not recognized on the basis of familiarity or because the target word failed to access the cue word during recognition.

Computer services were provided by the Computer Science Center at the University of Maryland. Requests for reprints should be sent to D. L. Horton, Department of Psychology, University of Maryland, College Park, MD 20742.
Although Rabinowitz et al.'s (1977) evidence is not particularly compelling, other evidence in support of their perspective was provided by Fisher (1979). In comparing recognition and recall as a function of cue-target relations, he found that cue-to-target associative strength affected only cued recall, whereas target-to-cue strength affected only recognition (cf. Hanley, 1984; Horton \& Mills, 1984).

The stimulus materials used in the experiments reported here were chosen on the basis of the preceding considerations. The word pairs presented at study varied in asymmetrical associative relatedness as well as in level of relatedness. When the dominant direction of associative relatedness went from cue to target, facilitation of cued recall but not of recognition was expected, and when the dominant direction of associative relatedness went from target to cue, facilitation of recognition but not of cued recall was expected. In other words, recognition failure was expected to occur when cue-to-target strength was dominant, but not to occur to any appreciable degree when target-to-cue strength was dominant.

In the recognition failure paradigm, target words can fall into one of four possible outcome categories. They can be remembered (both recognized and recalled), recognized only, recalled only, or forgotten (not recognized and not recalled). The recalled-only outcome represents recognition failure, the primary focus in this paper. Elsewhere, we have reported the results of our investigations of the other outcomes (Horton \& Pavlick, 1993; Horton, Pavlick, \& Moulin-Julian, 1993); we will draw on them only as is needed for interpretation of the recognition failure phenomenon. It should be noted, however, that we interpret the other outcomes as indicating that recognition consists of two processes-retrieval-based recognition and familiarity-based recognition. This is similar to Mandler's position, but we view the two processes as being inversely related to one another. In other words, they are opposing processes, which may not always be inversely related empirically but certainly are never complementary. 
We will discuss the implications of this interpretation for the phenomenon of recognition failure later in this paper.

\section{EXPERIMENTS 1-4}

The stimulus materials presented at study were identical in all four experiments, as were the conditions of presentation. In Experiments 1 and 2, for the recognition task, we used target words and an equal number of unrelated distractor items. In Experiments 3 and 4, we employed Diehl and Horton's (1988) related distractor task in recognition. For each target word, there were three distractor words associatively linked to both the cue and the target members of each pair. One other difference among these experiments was that in Experiments 2 and 4 an assessment of conscious recollection was required during recognition whenever a word was called old. In Experiments 1 and 3, this procedure was not employed.

\section{Method}

Materials. The stimulus materials were word pairs that were highly asymmetrical in associative strength according to a set of controlled association norms obtained from Douglas L. Nelson (personal communication, November 1983). Twenty-four word pairs, 8 at each of three levels of strength, were employed. The highest strength level $(\mathrm{H})$ ranged from .35 to .93 , with the average strength in the dominant direction being .57 and in the opposite direction .06 . An example is alarm-clock, which has an associative strength level of .39 in the alarm-to-clock direction and a .08 strength in the opposite direction. The intermediate strength level $(\mathrm{M})$ involved pairs ranging in strength from .21 to .28 , with an average strength in the dominant direction being .24 and in the opposite direction .02. An example is range-stove, which has an associative strength level of .21 in the range-to-stove direction and a .01 strength in the opposite direction. The lowest strength level (L) involved pairs ranging from .10 to .17 in strength, with an average strength in the dominant direction being .14 and in the opposite direction .01 . An example is bolt-lock, which has an associative strength level of .16 in the boltto-lock direction and a .02 strength in the opposite direction. For half the subjects, the dominant strength was from cue to target, and for the other half, the dominant strength was from target to cue. Dominant cueto-target strength was expected to facilitate cued recall but not recognition, whereas dominant target-to-cue strength was expected to facilitate recognition but not cued recall (Mandler, 1980). In further discussion, the direction of dominant strength will be referred to as path direction and the strength level as path strength.

Subjects. The subjects were undergraduate students who participated for course credit. The number of subjects in Experiments 1-4 were 40, 40,44 , and 64 . In each experiment, half the subjects were randomly assigned to each path direction condition.

\section{Results}

The data were analyzed in terms of the number of target words at each path strength level and path direction condition for each memory outcome. The results reported here are for the recalled-only outcome (i.e., recognition failure). The results for the other outcomes for Experiments 1 and 3 are reported by Horton and Pavlick (1993), and the results for the other outcomes for Experiments 2 and 4 are reported by Horton et al. (1993). For clarity of presentation, the results are reported as proportions. The recognition failure findings for all four experiments are reported in Table 1.

As can be seen in Table 1, performance in the cue-totarget path direction condition is substantially greater than
Table 1

Proportion of Targets in the Recalled-Only Outcome (Recognition Failure) in Each of the Four Experiments, as a Function of Path Direction and Path Strength

\begin{tabular}{ccccc}
\hline & \multicolumn{4}{c}{ Experiment } \\
\cline { 2 - 5 } Strength & 1 & 2 & 3 & 4 \\
\hline H & \multicolumn{4}{c}{ Cue to Target } \\
M & .21 & .21 & .25 & .28 \\
$\mathrm{~L}$ & .18 & .18 & .28 & .25 \\
& .19 & .14 & .15 & .19 \\
$\mathrm{H}$ & \multicolumn{4}{c}{ Target to Cue } \\
$\mathrm{M}$ & .03 & .05 & .07 & .08 \\
$\mathrm{~L}$ & .03 & .02 & .03 & .05 \\
& .03 & .04 & .05 & .05 \\
\hline
\end{tabular}

Note-H, high; M, medium; L, low.

performance in the target-to-cue path direction condition in all four experiments. Analysis of variance for the four experiments in order show $F(1,38)=36.43, p<.0001$; $F(1,38)=22.22, p<.0001 ; F(1,42)=31.68, p<$ .0001 ; and $F(1,62)=83.08, p<.0001$.

Inspection of Table 1 also suggests a path strength effect, particularly in the cue-to-target path direction condition. This observation is supported by a significant effect of path strength in both Experiment $3[F(2,84)=$ $3.33, p<.05]$ and Experiment $4[F(2,124)=6.01, p<$ .01 . In Experiment 3, there was also a significant interaction between path direction and path strength $[F(2,84)$ $=4.54, p<.02]$.

Although statistical comparisons were not carried out across experiments, inspection of Table 1 shows that the level of recognition failure was greater when the Diehl and Horton (1988) related distractor task was employed during recognition (Experiments 3 and 4). This finding is to be expected in view of the more difficult recognition task, and it is also consistent with the finding of greater recognition-only performance in these experiments (cf. Horton \& Pavlick, 1993; Horton et al., 1993).

\section{Discussion}

Before we discuss the recognition failure findings, it is appropriate to mention some other findings from the experiments reported by Horton et al. (1993) as well as by Horton and Pavlick (1993). In Experiments 1,3 , and 4 , there was a significant effect of path direction in recognition favoring the target-to-cue path direction condition. In Experiment 2 , this effect was marginally significant $(p=.06)$. In all four experiments, performance in the remembered outcome (targets both recognized and recalled) increased significantly with increases in path strength. However, performance in the recognized-only outcome increased significantly with decreases in path strength in all four experiments. The result of this inverse relationship between retrieval-based recognition (targets also recalled) and familiarity-based recognition (targets not recalled) is that overall recognition performance was not significantly related to path strength. Clearly, from the results just noted concerning the remembered outcome and the recognition failure results reported here, cued recall is strongly related to path strength.

The effects of path direction reported here for recognition failure and the effects of path direction on recognition hits noted previously strongly support the views of Mandler (1980) and those specifically stated by Hanley (1984), indicating that target-to-cue strength is very important for recognition. However, from the results reported by Horton et al. (1993) as well as by Horton and Pavlick (1993) it appears that increases 
in path strength for both path direction conditions lead to increases in retrieval-based recognition responses (targets also recalled), whereas decreases in path strength lead to increases in familiarity-based recognition responses (targets not recalled), in both path direction conditions.

According to Horton et al. (1993), retrieval-based recognition relies on the processes that influence both free and cued recall, and it involves episodic memory. In contrast, familiarity-based recognition operates in terms of a qualitatively different system involving sensory-perceptual features in semantic memory, much like the features involved in pattern recognition. It appears that increases in the quality of information in episodic memory (i.e., increases in path strength) lead to greater retrieval-based recognition and poorer familiarity-based recognition. In effect, increases in the quality of episodic information serve to inhibit familiarity-based recognition responses, the consequence being that overall recognition is not related to factors such as path strength.

This apparent digression into the nature of recognition memory is necessary if one is to comment on the frequently cited relationship between the probability of recognition and the probability of recognition failure (Tulving \& Wiseman, 1975). According to the Tulving-Wiseman function, if overall recognition does not vary with path strength, recognition failure should not vary with path strength; but, at least in our Experiments 3 and 4 , it does. Similar findings have also been reported by Vining and Nelson (1979, Experiment 1) as well as Bryant (1991, Experiment 2).

Yet it is simply not plausible to assert that a factor such as path strength, which significantly influences recall whether recognition is successful or not, has no significant effect on overall recognition performance. Recognition is not a unitary process; it involves two processes that frequently produce inversely related results. Therefore, predictions of the extent of recognition failure based on the Tulving-Wiseman (1975) function are fairly meaningless.

In this paper, we have reported effects of path direction and path strength on the incidence of recognition failure in four experiments. We have also shown, on the basis of the results reported by Horton and Pavlick (1993), and the results reported here, that predictions of recognition failure based on overall recognition performance are not particularly meaningful. The extent to which the Tulving-Wiseman (1975) function can be used to predict recognition failure probably arises because retrieval-based recognition responses from episodic memory are much more frequent than familiarity-based recognition responses from semantic memory. As we have seen, both retrieval-based recognition responses and level of recognition failure increase as path strength increases. Deviations from the Tulving-Wiseman function would be expected when familiarity-based recognition was high. This might well have been the case in studies like those of Begg (1979) and Gardiner and Tulving (1980), in which the cue and target words were not well integrated. In any case, the independence or dependence of recall and recognition is a nonissue.

\section{REFERENCES}

BEGG, I. (1979). Trace loss and the recognition failure of unrecalled words. Memory \& Cognition, 7, 113-123.

BRYANT, D. J. (1991). Exceptions to recognition failure as a function of the encoded association between cue and target. Memory \& Cognition, 19, 210-219.

Diehl, V. A., \& Horton, D. L. (1988). Encoding context effects in recognition and cued recall. Bulletin of the Psychonomic Society, 26, 393-394.

FishER, R. P. (1979). Retrieval operations in cued recall and recognition. Memory \& Cognition, 7, 224-231.

GaRDiner, J. M., \& TUlving, E. (1980). Exceptions to recognition failure of recallable words. Journal of Verbal Learning \& Verbal Behavior, 19, 194-209.

HANLEY, J. R. (1984). Dual processes in recognition and in recognition failure. Memory \& Cognition, 12, 575-580.

Horton, D. L., \& Mills, C. B. (1984). Human learning and memory. Annual Review of Psychology, 35, 361-394.

Horton, D. L., \& Pavlick, T. J. (1993). Associative relatedness effects in retrieval-based and familiarity-based recognition. Bulletin of the Psychonomic Society, 31, 19-22.

Horton, D. L., Pavlick, T. J., \& Moulin-Julian, M. W. (1993). Retrieval-based and familiarity-based recognition and the quality of information in episodic memory. Journal of Memory \& Language, 32, 39-55.

KINTSCH, W. (1970). Learning memory and conceptual processes. New York: Wiley.

MANDLER, G. (1980). Recognizing: The judgment of previous occurrence. Psychological Review, 87, 252-271.

Rabinowitz, J. C., Mandler, G., \& Barsalou, L. W. (1977). Recognition failure: Another case of retrieval failure. Journal of Verbal Learning \& Verbal Behavior, 16, 639-663.

Tulving, E. (1983). Elements of episodic memory. New York: Oxford University Press.

Tulving, E., \& Wiseman, S. (1975). Relation between recognition and recognition failure of recallable words. Bulletin of the Psychonomic Society, 6, 79-82.

Vining, S. K., \& NeLson, T. O. (1979). Some constraints on the generality and interpretation of the recognition failure of recallable words. American Journal of Psychology, 92, 257-276.

(Manuscript received June 1, 1993.) 